\title{
Risk Factor Assessment for Pre-eclampsia: A Case Control Study
}

\author{
Manoj Kumar Verma, Priyanka Kapoor, ${ }^{*}$ Rajeev Yadav, Ravindra Kumar Manohar
}

\begin{abstract}
Background: Knowledge and identification of risk factors for preeclampsia will help in estimating each woman's individualized risk and possibly reduce the recurrence risk of preeclampsia. So this study was conducted to assess and compare the socio-demographic profile of women with and without preeclampsia and to determine the risk factors associated with preeclampsia. Methodology: This Hospital based Case control analytical study was conducted among 180 preeclampsia case and 180 control subjects at Mahila chikitsalaya, Jaipur, from June 2014 to May 2015. Results: Bivariate analysis found that preeclampsia was significantly associated with rural residence $(p=0.033)$, joint family type $(p=0.025)$, low education of head of family $(p=0.007)$, young age at menarche (11-12years) $(p<0.001)$ Anemia $(P=0.034)$ and primiparity $(p<0.001)$, Family history of preeclampsia $(p<0.001)$ and hypertension $(p=0.007)$ and Non veg. diet $(p=0.042)$. Preeclampsia was not found to be significantly associated with history of previous abortion, inter pregnancy period and sex of last child of multiparous women and ANC characteristics, TT immunization, IFA tablets, twin pregnancy or gestational diabetes. In the multiple regression analysis, Age $>30$ years, Preobese $(\mathrm{BMl}=25-29.9)$, obese $(\mathrm{BMl} \geq 30 \mathrm{Kg} /$ $\mathrm{m} 2)$, Primiparity, age of menarche at 12 years and rural residence were found to be independent risk factors associated with Preeclampsia. Conclusion: Age $>30$ years, Preobese, Obese, Primiparity, early age of menarche (12 years) and Rural residence were found as significant predictor for development of Preeclampsia. Most of these factors are non modifiable, but can be used to screen women during antenatal visits to identify those at higher risk of Preeclampsia. Key words: Preeclampsia, Risk Factor, Pregnancy.
\end{abstract}

\section{Manoj Kumar Verma, Priyanka Kapoor,* Ra- jeev Yadav, Ravindra Kumar Manohar \\ M.B.B.S.M.D. (Community Medicine) Senior Resident Department of Community Medicine S.M.S. Medical College, Jaipur (Rajasthan) INDIA.}

\section{Correspondence} Priyanka Kapoor

Senior Resident, Department of Community Medicine, S.M.S. Medical College, Jaipur (Rajasthan), INDIA.

Mobile no: 9414954560

Email: priyankakapoor19@gmail.com

History

- Submission Date: 25-12-2016;

- Revised Date: 09-04-2017;

- Accepted Date: 17-07-2017.

DOI : 10.5530/ijmedph.2017.3.35

Article Available online

http://www.ijmedph.org/v7/i3

\section{Copyright}

(C) 2017 Phcog.Net. This is an openaccess article distributed under the terms of the Creative Commons Attribution 4.0 International license.

\section{INTRODUCTION}

Hypertensive disorders of pregnancy are one of the most common causes of maternal and fetal morbidity and mortality accounting for more than 40,000 maternal deaths worldwide annually. ${ }^{1}$ In developing countries, it causes about one third of maternal mortality. ${ }^{2}$ India accounted for $19 \%$ of maternal deaths worldwide. ${ }^{3}$ Five percent of maternal deaths in India are due to Hypertensive disorder. ${ }^{4}$

Preeclampsia (PE) has remained a significant public health threat in both developed and developing countries, ${ }^{5}$ however, the impact of the disease is felt more severely in developing countries, where treatment may be ineffective due to late presentation of cases. Although pre-eclampsia is not a totally preventable condition, its early detection and proper treatment can prevent severity, ${ }^{6}$ Unclear etiology and the unpredictable nature of the disease further worsen the situation. The incidence of $\mathrm{PE}$ ranges from $2 \%$ to $10 \% .^{4-7}$ WHO estimates the incidence of preeclampsia to be seven times higher in developing countries $(2.8 \%$ of live births) than in developed countries $(0.4 \%) .{ }^{8}$ Preeclampsia has been associated with increased risk of adverse fetal, neonatal and maternal outcomes including Antepartum and Postpartum haemorrhage, Acute renal and Hepatic failure, eclamptic seizures, stroke, Placental abruption, HELLP syndrome Heart

failure, DIC, Multiple organ failures and Maternal death. ${ }^{9-12}$ Fetal complications include Fetal distress, Intrauterine growth retardation, Preterm birth, Stillbirth, Perinatal death and Neonatal asphyxia. ${ }^{11-15}$ The factors that have been postulated to influence the risk of pre-eclampsia in various studies include Diabetes and Gestational Diabetes, ${ }^{12-14-16-18}$ Obesity, ${ }^{19}$ Multiple pregnancy. ${ }^{17,18}$ Personal and family history of pre-eclampsia, ${ }^{20}$ chronic infections, ${ }^{21} \mathrm{UTI},{ }^{2}$ First pregnancy and Older maternal age or Younger than 20 years age, ${ }^{2}$ Renal disease and autoimmune disorder, ${ }^{16,17}$ prolonged interval between pregnancies ${ }^{2-18}$ and History of abortion ${ }^{8}$ and maternal diet. ${ }^{22}$

Rationale: Knowledge and identification of sociodemographic and clinical risk factors for PE will help in estimating each woman's individualized risk and allow antenatal surveillance to be directed at these women and guide the healthcare providers for counseling of such women and possibly reduce the recurrence risk of $\mathrm{PE}$ if some modifiable risk factors (like obesity) are present.

While most studies have been undertaken in high-income settings, some inconsistencies exist, especially in developing settings where pre-eclampsia risk factors have been explored less. Furthermore, conditions such as severe anaemia and lack of antenatal care that 
are more prevalent in less developed regions require further investigation and validation of findings.

Aims and objectives were to assess and compare the socio-demographic profile of women with and without preeclampsia and to determine the risk factors associated with preeclampsia.

\section{METHODOLOGY}

This Hospital based Case control analytical study was conducted at Mahila chikitsalaya, Jaipur, from June 2014 to May 2015. 180 preeclampsia case and 180 control subjects were included in the study. Sample size was calculated at alpha error 0.05 and power $80 \%$, taking the expected Odds ratio for family History of Hypertension as a risk factor of preeclampsia as 5.48 and proportion of control subjects with Family History of Hypertension as $0.02 .^{23}$

Case group: Woman admitted to post natal ward who delivered during preceding 2 days, who in the antenatal period or before going to labor was diagnosed by a doctor as having preeclampsia.

Control Group: Women admitted to post natal ward who delivered during preceding 2 days and did not had Preeclampsia during pregnancy.

Pre-eclampsia (ICD-10 code 014) was defined as a diastolic blood pressure of at least $90 \mathrm{mmHg}$ on two or more consecutive occasions 24 hours apart or a diastolic blood pressure of at least $110 \mathrm{mmHg}$ on any one occasion plus proteinuria (one 24-hour urine collection with a total protein excretion of at least $300 \mathrm{mg}$ or two $1+$ on a urine dipstick). ${ }^{24}$

Study subjects were selected from three post Natal wards of Mahila Chikitsalaya, Jaipur after getting ethical clearance from research review board. Each day all delivered women fulfilling the criteria for Preeclampsia case were enrolled into the study. Same no of controls were then selected each day by random selection of remaining beds. Case and control were enrolled within 2 days of delivery and informed consent was taken. Matching among cases and controls was not performed because many of the socio-demographic parameters are suspected risk factors for PE. Information was collected from hospital case sheet of the women and by interviewing the Study subject herself. In case, a patient was comatose after delivery, the history was taken when she regained consciousness or from relatives who accompanied her. Women, who delivered before 20 week of gestation irrespective of the outcome, were excluded from study. Anaemia was defined as Hemoglobin $<11 \mathrm{~g} / \mathrm{dl}$. A women was considered non vegetarian if she took any non vegetarian dish (excluding egg), at least once a week in the last one month.

All the information was collected using a Predesigned, semi-structured study Performa to eliminate recording bias. Potential risk factors for study were selected on the basis of literature review and biological plausibility for an association with both the exposure and outcome.

Statistical analysis: Data obtained from study Performa was entered in MS Excel to figure Master Chart. Continuous variables were summarized as mean and standard deviation while categorical variables were summarized as proportion (\%). Unpaired t-test was used for comparison of continuous variable while Chi-square test was used for analysis of Categorical variable. Risk was assessed by calculation of Odds Ratio in Bivariate analysis. Step wise Multiple Logistic Regression analysis was done to find out predictors of Preeclampsia. All variables, found significantly associated with Preeclampsia, were entered in Regression model. Probability of independent variable in retaining Regression model was kept $<0.05$ while that of removal as $>0.10$. All statistical calculations were done by using Med Calc.14.2.1.0 software. $\mathrm{P}<0.05$ was taken as significant.

\section{RESULT}

Present study included 180 preeclampsia cases and 180 controls. Most of the study population belonged to 21-30 years of age. More preeclampsia women (25\%) belonged to $\leq 20$ years as compared to controls (17.8\%). Both groups did not differ significantly in relation to education, religion, occupation and socio-economic status. Bivariate analysis found that preeclampsia was significantly associated with rural residence $(p=0.033)$, joint family type $(p=0.025)$, low education of head of family $(p=0.007)$, primiparity $(p<0.001)$, young age at menarche $(11-12$ years $)(p<0.001)$, Anemia $(\mathrm{P}=0.034)$, Family history of preeclampsia $(\mathrm{p}<0.001)$ and hypertension $(\mathrm{p}=0.007)$ and Non veg. $\operatorname{diet}(\mathrm{p}=0.042)$. (Table 1-4). There was 1.22 times more risk in joint family in bivariate analysis. Similarly illiterate, middle and graduate mothers were having 1.05, 1.30 and 1.25 times more risk. 1.33 times more risk was found when the age at conception was between 21 to 25 years. Rh positive mothers were having 2.7 times more risk and mothers having family history of hypertension were found to be at 2.8 times more risk of developing preeclampsia. Likewise non vegetarian mothers have 1.63 times risk of preeclampsia.

Young age at menarche (11-12 years) was significantly more among preeclampsia (46\%) as compared to controls (23\%). Women with menarche at $11 \& 12$ years had more than thrice the risk of preeclampsia as compared to those with menarche at 14 years. Preeclampsia was also significantly associated with primiparity. The risk of preeclampsia in primipara was more than twice $(\mathrm{OR}=2.39 ; 1.23-4.65)$ as compared to those with third parity. Preeclampsia was not found to be significantly associated with history of previous abortion, inter pregnancy period and sex of last child of multiparous women (Table-2). Anaemia was found to be associated with preeclampsia $(\mathrm{P}=0.034)$, however no association was found between preeclampsia and ANC characteristics, TT immunization, IFA tablets, twin pregnancy or gestational diabetes (Table-3). Personal history of preeclampsia and Family history of hypertension were also significantly associated with preeclampsia (table 4). Non veg. diet was found to be significantly more among Preeclampsia cases (37.2\%) as compared to controls (26.7\%) (Table-4). Non vegetarian had odds of Preeclampsia 1.6 times than that in vegetarians. Smoking and tobacco chewing was not found to be associated with preecmlampsia.

The variables that were found to be significant in bivariate analysis were then entered in the regression model for stepwise multiple regression analysis. Age $>30$ years, Preobese $(\mathrm{BMI}=25-29.9)$, obese $(\mathrm{BMI} \geq 30 \mathrm{Kg}$ / $\mathrm{m} 2$ ), Primiparity, age of menarche at 12 years and rural residence were found to be independent risk factors associated with Preeclampsia (table-5).

On applying multiple logistic regression, patients with age $>30$ years were found to have 2.87 times risk of preeclamsia as compared to age $<30$ years. Primipara mothers had 4.5 times risk as compared to multipara mothers. As compared to normal and underweight, preobese and obese were having 3.23 times and 8.28 times more risk of PE respectively. Risk of Preeclampsia was 2.59 times high in patients with age of menarche $<12$ years as compared to those in which age $>12$ years. Similarly rural residents were having 1.67 times higher risk for preeclampsia as compared to urban residents.

\section{DISCUSSION}

Present study aimed to determine the socio-demographic and clinical factors that increase risk of Preeclampsia. Though, many socio-demographic factors were found to be associated with Preeclampsia in bivariate analysis, multiple logistic regression analysis selected out the independent risk factors for Preeclampsia.

Maternal age $>30$ years was found to increase the risk of Preeclampsia $(\mathrm{OR}=2.87 ; 1.03-7.99)$. Various studies..$^{8-25-26-28}$ have also reported advanced maternal age $>30 / 35$ years to be associated with Preeclampsia. Increased age of women is an important risk factor probably due to increased villous reaction leading to pre-eclampsia in a woman greater 


\begin{tabular}{|c|c|c|c|c|c|c|c|c|c|}
\hline \multirow{2}{*}{\multicolumn{2}{|c|}{$\begin{array}{l}\text { Socio-demographic characteristics } \\
\text { No. }\end{array}$}} & \multicolumn{2}{|c|}{ Case } & \multicolumn{2}{|c|}{ Control } & \multicolumn{2}{|c|}{ Total } & \multirow{2}{*}{$\begin{array}{l}\text { Odds ratio } \\
(95 \% \mathrm{CL})\end{array}$} & \multirow{2}{*}{$P$ value } \\
\hline & & $\%$ & No. & $\%$ & No. & $\%$ & & & \\
\hline \multirow{4}{*}{ Age Group (years) } & $\leq 20$ & 45 & 25.0 & 19 & 10.6 & 64 & 17.8 & $1.547(0.977-2.45)$ & \multirow{4}{*}{$<0.001$} \\
\hline & $21-25$ & 85 & 47.2 & 102 & 56.7 & 187 & 51.9 & - & \\
\hline & $26-30$ & 36 & 20.0 & 52 & 28.9 & 88 & 24.4 & $0.831(0.497-1.38)$ & \\
\hline & $>30$ & 14 & 7.8 & 7 & 3.9 & 21 & 5.8 & $2.400(0.92-6.217)$ & \\
\hline \multirow{3}{*}{ Religion } & Hindu & 137 & 76.1 & 138 & 76.7 & 275 & 76.4 & $0.970(0.59-1.577)$ & \multirow{2}{*}{1.000} \\
\hline & Muslim & 43 & 23.8 & 42 & 23.3 & 85 & 23.6 & - & \\
\hline & Urban & 56 & 31.1 & 80 & 44.4 & 136 & 37.8 & - & \multirow{3}{*}{0.033} \\
\hline \multirow[t]{2}{*}{ Residence } & Rural & 113 & 62.8 & 91 & 50.6 & 204 & 56.7 & $1.774(1.143-2.75)$ & \\
\hline & Slum & 11 & 6.1 & 9 & 5.0 & 20 & 5.6 & $1.746(0.679-4.49)$ & \\
\hline \multirow{3}{*}{ Family Type } & Nuclear & 18 & 10.0 & 36 & 20.0 & 54 & 15.0 & $0.53(0.219-1.289)$ & \multirow{3}{*}{0.025} \\
\hline & Joint & 146 & 81.1 & 127 & 70.6 & 273 & 75.8 & $1.221(0.59-2.517)$ & \\
\hline & 3 generation & 16 & 8.9 & 17 & 9.4 & 33 & 9.2 & - & \\
\hline \multirow{6}{*}{ Education } & Illiterate & 70 & 38.9 & 65 & 36.1 & 135 & 37.5 & $1.533(0.837-2.81)$ & \multirow{6}{*}{0.358} \\
\hline & Primary & 26 & 14.4 & 24 & 13.3 & 50 & 13.9 & $1.542(0.73-3.257)$ & \\
\hline & Middle & 21 & 11.7 & 15 & 8.3 & 36 & 10.0 & $1.992(0.868-4.57)$ & \\
\hline & High & 26 & 14.4 & 37 & 20.5 & 63 & 17.5 & - & \\
\hline & Post High school & 14 & 7.78 & 22 & 12.2 & 36 & 10.0 & $0.906(0.39-2.091)$ & \\
\hline & Graduation /PG & 23 & 12.8 & 17 & 9.44 & 40 & 11.1 & $1.925(0.86-4.297)$ & \\
\hline \multirow{2}{*}{ Occupation } & House Wife & 160 & 88.9 & 161 & 89.4 & 321 & 89.2 & $0.944(0.486-1.84)$ & \multirow{2}{*}{1.000} \\
\hline & Working & 20 & 11.1 & 19 & 10.6 & 39 & 10.8 & - & \\
\hline \multirow{7}{*}{$\begin{array}{l}\text { Education Head of } \\
\text { family }\end{array}$} & Illiterate & 53 & 29.4 & 57 & 31.7 & 110 & 30.6 & $1.051(0.536-2.06)$ & \multirow{7}{*}{0.007} \\
\hline & Primary & 45 & 25.0 & 24 & 13.3 & 69 & 19.2 & $2.120(1.003-4.480)$ & \\
\hline & Middle & 22 & 12.2 & 19 & 10.6 & 41 & 11.4 & $1.309(0.57-3.007)$ & \\
\hline & High & 23 & 12.8 & 26 & 14.4 & 49 & 13.6 & - & \\
\hline & Post $\mathrm{H}$ & 15 & 8.33 & 36 & 20.0 & 51 & 14.2 & $0.471(0.207-1.07)$ & \\
\hline & Graduate & 20 & 11.1 & 18 & 10. & 38 & 10.6 & $1.256(0.538-2.94)$ & \\
\hline & Honors' & 2 & 1.11 & 0 & 0.0 & 2 & 0.6 & NA & \\
\hline \multirow{4}{*}{ Socio-economic status } & I & 4 & 2.2 & 4 & 2.22 & 8 & 2.22 & $1.044(0.246-4.43)$ & \multirow{4}{*}{1.000} \\
\hline & II & 85 & 47.2 & 76 & 42.2 & 161 & 44.7 & $1.168(0.70-1.951)$ & \\
\hline & III & 45 & 25 & 47 & 26.1 & 92 & 25.6 & -- & \\
\hline & IV & 46 & 25.6 & 53 & 29.4 & 99 & 27.5 & $0.906(0.513-1.60)$ & \\
\hline
\end{tabular}

than 30years, as has been found in other studies. ${ }^{13-15}$ Age of menarche at 12 years was found to be a significant risk factor for Preeclampsia $\left(\mathrm{OR}=2.59\right.$; 1.53-4.40), similar to finding of Ramesh et al ${ }^{4}$ (2014). Early menarche $(\leq 12 y)$ has been associated with increased risk of CVD events in other studies potentially mediated by increased adiposity associated with early menarche. ${ }^{25}$ Present study found Prim parity to be an independent risk factor for development of Preeclampsia ( $O R=4.51 ; 2.76$ 7.36) as has been found in many studies. ${ }^{24,25-27-32-33}$ This is because prim parity is due to initial trophoblastic invasion and how the mother reacts to it. The failure of the normal invasion of trophoblastic cells leads to maladaptation of the spiral arterioles, which are related to the causation of pre-eclampsia. ${ }^{34}$ In this study, Rural Residence was found to a significant risk factor for development of Preeclampsia. This correlated with the findings of a national cross sectional study (NFHS-05-06) by Sutapa Agrawal et al..$^{35}$ In present study, BMI 25-29.9 Kg/m² (Preobese; $\mathrm{OR}=3.23 ; 1.66-6.29$ ), and $\mathrm{BMI} \geq 30 \mathrm{Kg} / \mathrm{m}^{2}$ (obese; $\mathrm{OR}=8.28 ; 2.89-27.55$ ) were found to be independent risk factors for Preeclampsia. Overweight and obesity have been reported as significant risk factor by many studies. $^{7-13-17,18-25,26-33}$ 


\begin{tabular}{|c|c|c|c|c|c|c|c|c|c|}
\hline \multirow{2}{*}{\multicolumn{2}{|c|}{$\begin{array}{l}\text { Obstetric characteristics } \\
\text { No. }\end{array}$}} & \multicolumn{2}{|c|}{ Case } & \multicolumn{2}{|c|}{ Control } & \multicolumn{2}{|c|}{ Total } & \multirow{2}{*}{$\begin{array}{l}\text { Odds ratio } \\
(95 \% \mathrm{CL})\end{array}$} & \multirow{2}{*}{$P$ value } \\
\hline & & $\%$ & No. & $\%$ & No. & $\%$ & & & \\
\hline \multirow{5}{*}{$\begin{array}{c}\text { Age at Menarche } \\
\text { (years) }\end{array}$} & 11 & 10 & 5.6 & 4 & 2.2 & 14 & 3.9 & $4.53(1.222-16.800)$ & \multirow{5}{*}{$<0.001$} \\
\hline & 12 & 73 & 40.6 & 38 & 21.1 & 111 & 30.8 & $3.482(1.686-7.192)$ & \\
\hline & 13 & 79 & 43.9 & 98 & 54.4 & 177 & 49.2 & $1.461(0.741-2.88)$ & \\
\hline & 14 & 16 & 8.9 & 39 & 21.7 & 55 & 15.3 & - & \\
\hline & 15 & 2 & 1.1 & 1 & 0.6 & 3 & 0.8 & $3.625(0.305-3.153)$ & \\
\hline \multirow{5}{*}{ Parity } & 1 & 117 & 65.0 & 56 & 31.1 & 173 & 48.1 & $2.388(1.226-4.651)$ & \multirow{5}{*}{$<0.001$} \\
\hline & 2 & 30 & 16.7 & 81 & 45.0 & 111 & 30.8 & $0.423(0.206-0.870)$ & \\
\hline & 3 & 21 & 11.7 & 24 & 13.3 & 45 & 12.5 & - & \\
\hline & 4 & 8 & 4.4 & 14 & 7.8 & 22 & 6.1 & $0.653(0.229-1.86)$ & \\
\hline & 5 & 4 & 2.2 & 5 & 2.8 & 9 & 2.5 & $0.914(0.217-3.856)$ & \\
\hline \multirow{3}{*}{$\begin{array}{l}\text { Age at } 1^{\mathrm{ST}} \\
\text { Conception }\end{array}$} & $\leq \mathbf{2 0}$ & 103 & 57.2 & 118 & 65.6 & 221 & 61.4 & -- & \multirow{3}{*}{0.034} \\
\hline & $21-25$ & 72 & 40.0 & 62 & 34.4 & 134 & 37.2 & $1.330(0.865-2.046)$ & \\
\hline & $>25$ & 5 & 2.8 & 0 & 0.0 & 5 & 1.4 & NA & \\
\hline \multirow{2}{*}{ previous Abortion } & No & 37 & 20.6 & 31 & 17.3 & 68 & 18.9 & $1.244(0.732-2.112)$ & \multirow{2}{*}{0.501} \\
\hline & Yes & 143 & 79.4 & 149 & 82.7 & 292 & 81.1 & - & \\
\hline \multirow{2}{*}{$\begin{array}{l}\text { Inter pregnancy } \\
\text { period }\end{array}$} & $<3$ years & 36 & 57.2 & 79 & 63.7 & 115 & 61.5 & $0.759(0.409-1.410)$ & \multirow{2}{*}{0.476} \\
\hline & $\geq 3$ years & 27 & 42.9 & 45 & 36.3 & 72 & 38.5 & - & \\
\hline \multirow{2}{*}{ Sex of Last Child } & Male & 28 & 44.4 & 55 & 44.5 & 83 & 28 & $1.004(0.545-1.848)$ & \multirow{2}{*}{0.885} \\
\hline & Female & 35 & 55.6 & 69 & 55.6 & 104 & 35 & - & \\
\hline
\end{tabular}

\begin{tabular}{|c|c|c|c|c|c|c|c|c|c|}
\hline \multirow{2}{*}{\multicolumn{2}{|c|}{$\begin{array}{l}\text { ANC characteristics } \\
\text { No. }\end{array}$}} & \multicolumn{2}{|c|}{ Case } & \multicolumn{2}{|c|}{ Control } & \multirow{2}{*}{\multicolumn{2}{|c|}{$\%$ Total }} & \multirow{3}{*}{$\begin{array}{c}\text { Odds ratio } \\
(95 \% \mathrm{CL}) \\
0.956(0.63-1.448)\end{array}$} & \multirow{2}{*}{$P$ value } \\
\hline & & $\%$ & No. & $\%$ & No. & & & & \\
\hline \multirow{2}{*}{ Completion of ANC } & No & 81 & 45 & 83 & 46.1 & 164 & 45.6 & & \multirow{2}{*}{0.916} \\
\hline & Yes & 99 & 55 & 97 & 53.9 & 196 & 54.4 & - & \\
\hline \multirow{3}{*}{$\begin{array}{c}\text { Trimester of } 1^{\text {st }} \text { ANC } \\
\text { visit }\end{array}$} & 1 & 74 & 41.1 & 74 & 41.1 & 148 & 41.1 & - & \multirow{3}{*}{0.948} \\
\hline & 2 & 88 & 48.9 & 94 & 52.2 & 182 & 50.6 & $0.936(0.607-1.45)$ & \\
\hline & 3 & 10 & 5.6 & 11 & 6.1 & 21 & 5.8 & $0.909(0.364-2.27)$ & \\
\hline \multirow{3}{*}{ TT immunization } & Incomplete & 4 & 2.22 & 0 & 0.00 & 4 & 1.11 & - & \multirow{2}{*}{0.131} \\
\hline & Complete & 176 & 97.8 & 180 & 100 & 356 & 98.9 & - & \\
\hline & Not taken & 23 & 12.8 & 17 & 9.44 & 40 & 11.1 & $1.207(0.55-2.640)$ & \multirow{4}{*}{0.218} \\
\hline \multirow{3}{*}{ IFA tablets taken } & $<50$ & 67 & 37.2 & 57 & 31.7 & 124 & 34.4 & $1.048(0.58-1.886)$ & \\
\hline & $50-100$ & 53 & 29.4 & 73 & 40.6 & 126 & 35.0 & $0.648(0.36-1.165)$ & \\
\hline & 100 & 37 & 20.6 & 33 & 18.3 & 70 & 19.4 & - & \\
\hline \multirow{2}{*}{$\begin{array}{l}\text { Gestational Diabetes } \\
\qquad(\mathrm{N}=135)\end{array}$} & No & 18 & 20.7 & 7 & 14.6 & 25 & 18.5 & $1.528(0.588-3.97)$ & \multirow{2}{*}{0.520} \\
\hline & Yes & 69 & 79.3 & 41 & 85.4 & 110 & 81.5 & - & \\
\hline \multirow{2}{*}{ Gestation } & Single & 172 & 95.6 & 169 & 93.9 & 341 & 94.7 & - & \multirow[t]{2}{*}{0.637} \\
\hline & Twin & 8 & 4.4 & 11 & 6.1 & 19 & 5.3 & $0.715(0.280-1.82)$ & \\
\hline \multirow{4}{*}{ Hemoglobin level } & Normal & 35 & 19.4 & 18 & 10 & 53 & 14.7 & - & \multirow{4}{*}{0.034} \\
\hline & Mild Anaemia & 19 & 10.6 & 30 & 16.7 & 49 & 13.6 & $0.326(0.15-0.73)$ & \\
\hline & Moderate Anaemia & 107 & 59.4 & 118 & 65.6 & 225 & 62.5 & $0.466(0.249-0.87)$ & \\
\hline & Severe Anaemia & 19 & 10.6 & 14 & 7.8 & 33 & 9.2 & $0.698(0.285-1.71)$ & \\
\hline
\end{tabular}




\begin{tabular}{|c|c|c|c|c|c|c|c|c|c|}
\hline & & \multicolumn{2}{|c|}{ Case } & \multicolumn{2}{|c|}{ Control } & \multicolumn{2}{|c|}{ Total } & \multirow{2}{*}{$\begin{array}{c}\text { Odds ratio } \\
(95 \% \mathrm{CL})\end{array}$} & \multirow{2}{*}{$P$ value } \\
\hline & & No. & $\%$ & No. & $\%$ & No. & $\%$ & & \\
\hline \multirow{4}{*}{ BMI } & Under weight & 17 & 9.4 & 20 & 11.1 & 37 & 10.3 & $1.133(0.565-2.27)$ & \multirow{4}{*}{$<0.001$} \\
\hline & Normal & 102 & 56.7 & 136 & 75.6 & 238 & 66.1 & -- & \\
\hline & Preobese & 41 & 22.8 & 20 & 11.1 & 61 & 16.9 & $2.733(1.51-4.946)$ & \\
\hline & Obese & 20 & 11.1 & 4 & 2.2 & 24 & 6.7 & $6.667(2.211-20.1)$ & \\
\hline \multirow{4}{*}{ Blood Group } & A & 22 & 15.8 & 28 & 21.1 & 50 & 18.4 & $0.818(0.41-1.618)$ & \multirow{4}{*}{0.284} \\
\hline & $\mathrm{AB}$ & 20 & 14.4 & 23 & 17.3 & 43 & 15.8 & $0.905(0.44-1.852)$ & \\
\hline & B & 49 & 35.3 & 51 & 38.4 & 100 & 36.8 & - & \\
\hline & O & 48 & 34.5 & 31 & 23.3 & 79 & 29 & $1.612(0.886-2.93)$ & \\
\hline \multirow{3}{*}{ Rh factor } & Positive & 131 & 94.2 & 114 & 85.7 & 245 & 90.1 & $2.729(1.15-6.471)$ & \multirow{2}{*}{0.032} \\
\hline & Negative & 8 & 5.8 & 19 & 14.3 & 27 & 9.9 & - & \\
\hline & Preeclampsia $^{1}$ & 18 & 28.6 & 0 & 0.0 & 18 & 9.6 & - & $<0.001$ \\
\hline \multirow{4}{*}{$\begin{array}{l}\text { personal } \\
\text { History of }\end{array}$} & Hypertension & 0 & & 0 & & 0 & & - & \multirow{4}{*}{1.000} \\
\hline & Diabetes Mellitus & 1 & 0.6 & 0 & & 1 & 0.3 & - & \\
\hline & Renal Disease & $\mathbf{0}$ & & 0 & & 0 & & - & \\
\hline & CVD & 0 & & 0 & & 0 & & - & \\
\hline \multirow{2}{*}{$\begin{array}{l}\text { Family } \\
\text { History }\end{array}$} & Preeclampsia & 1 & 0.6 & 0 & & 1 & 0.3 & NA & $<0.001$ \\
\hline & Hypertension & 40 & 22.2 & 20 & 11.1 & 60 & 16.7 & $2.28(1.27-4.09)$ & 0.007 \\
\hline \multirow{2}{*}{ Diet } & Non Vegetarian & 67 & 37.2 & 48 & 26.7 & 115 & 31.9 & $1.631(1.042-2.551)$ & \multirow{2}{*}{0.042} \\
\hline & Vegetarian & 113 & 62.8 & 132 & 73.3 & 245 & 68.1 & - & \\
\hline
\end{tabular}

${ }^{1 \cdot}$ In Multiparous women

Table 5: Multiple Logistic Regression analysis

\begin{tabular}{|c|c|c|c|c|c|}
\hline Variable & Coefficient & $\begin{array}{l}\text { Std. } \\
\text { Error }\end{array}$ & $\begin{array}{l}\text { Odds } \\
\text { ratio }\end{array}$ & $95 \% \mathrm{Cl}$ & P value \\
\hline $\begin{array}{c}\text { Age }<30 \text { years } \\
\text { (Reference category) }\end{array}$ & - & - & - & - & - \\
\hline Age $>30$ years & 1.05429 & 0.52293 & 2.8699 & $1.0298-7.9984$ & 0.0438 \\
\hline Normal and underweight (Reference category) & - & - & - & - & - \\
\hline $\begin{array}{c}\text { Preobese } \\
\left(\text { BMI } 25-29.9 \mathrm{~kg} / \mathrm{m}^{2}\right)\end{array}$ & 1.17387 & 0.33953 & 3.2345 & $1.6626-6.2925$ & 0.0005 \\
\hline $\begin{array}{c}\text { Obese } \\
\left(\mathrm{BMI} \geq 30 \mathrm{Kg} / \mathrm{m}^{2}\right)\end{array}$ & 2.11400 & 0.61334 & 8.2813 & 2.4889-27.5537 & 0.0006 \\
\hline $\begin{array}{c}\text { Multiparity } \\
\text { (Reference category) }\end{array}$ & - & - & - & - & - \\
\hline Primiparity & 1.50664 & 0.24977 & 4.5116 & 2.7652-7.3609 & $<0.0001$ \\
\hline $\begin{array}{c}\text { Age of menarche } \\
\quad>12 \text { years } \\
\text { (Reference category) }\end{array}$ & - & - & - & - & - \\
\hline $\begin{array}{l}\text { Age of menarche } \\
\quad<12 \text { years }\end{array}$ & 0.95307 & 0.26933 & 2.5937 & $1.5299-4.3971$ & 0.0004 \\
\hline $\begin{array}{c}\text { Urban residence } \\
\text { (Reference category) }\end{array}$ & - & - & - & - & - \\
\hline Rural residence & 0.51211 & 0.25004 & 1.6688 & $1.0223-2.7242$ & 0.0405 \\
\hline Constant & -1.6805 & & & & \\
\hline
\end{tabular}




\section{CONCLUSION}

Age $>30$ years, Preobese, Obese, Primiparity, early age of menarche (12 years) and Rural residence as significant predictor for development of Preeclampsia. Most of these factors are non-modifiable, but can be used to screen women dursing antenatal visits to identify those at higher risk of Preeclampsia.

\section{CONFLICT OF INTEREST}

None declared

\section{REFERENCES}

1. Imdad A, Jabeen A, Bhutta ZA. Role of calcium supplementation during pregnancy in reducing risk of developing gestational hypertensive disorders: a meta-analysis of studies from developing countries. BMC public health. 2011;11(3):S18.

2. Petla LT, Chikkala R, Ratnakar KS, Kodati V, Sritharan V. Biomarkers for the management of pre-eclampsia in pregnant women. The Indian journal of medical research. 2013;138(1):60.

3. A Strategic Approach to Reporoductive, Maternal, Newborn, Child And Adolescent Health (Rmnch+A) In India. New Delhi: Ministry of Health \& Family Welfare Government of India; January, 2013. 04 p. Available from: http://cghealth. nic.in/ehealth/2016/RMNCH/1_RMNCHA_Strategy.pdf [Assessed on May 12th 2016]

4. Ramesh K, Gandhi S, Rao V. Socio-demographic and other risk factors of preeclampsia at a tertiary care hospital, karnataka: case control study. Journal of clinical and diagnostic research: JCDR. 2014;8(9):JC01.

5. Surapaneni T, Bada VP, Nirmalan CP. Risk for recurrence of pre-eclampsia in the subsequent pregnancy. Journal of clinical and diagnostic research: JCDR. 2013;7(12):2889.

6. Manandhar BL, Chongstuvivatwong V, Geater A. Antenatal care and severe pre-eclampsia in Kathmandu valley. Journal of Chitwan Medical College. 2014;3(4):43-7.

7. Bej P, Chhabra P, Sharma AK, Guleria K. Determination of risk factors for preeclampsia and eclampsia in a tertiary hospital of India: a case control study. Journal of family medicine and primary care. 2013;2(4):371.

8. Endeshaw M, Ambaw F, Aragaw A, Ayalew A. Effect of maternal nutrition and dietary habits on preeclampsia: A case-control study. International Journal of Clinical Medicine. 2014;5(21):1405-16.

9. North RA, McCowan LM, Dekker GA, Poston L, Chan EH, Stewart AW, et al. Clinical risk prediction for pre-eclampsia in nulliparous women: development of model in international prospective cohort. Bmj. 2011;342:d1875.

10. Ye C, Ruan Y, Zou L, Li G, Li C, Chen Y, et al. The 2011 survey on hypertensive disorders of pregnancy (HDP) in China: prevalence, risk factors, complications, pregnancy and perinatal outcomes. PloS one. 2014;9(6):e100180.

11. Kayode O. Osungbade and Olusimbo K. Ige. Public Health Perspectives of Preeclampsia in Developing Countries: Implication for Health System Strengthening. Hindawi Publishing Corporation Journal of Pregnancy.2011; Volume 2011,ArticleID 481095,6 pages doi:10.1155/2011/481095

12. Roberts CL, Ford JB, Algert CS, Antonsen S, Chalmers J, Cnattingius S, et al. Population-based trends in pregnancy hypertension and pre-eclampsia: an international comparative study. BMJ open. 2011;1(1):e000101.

13. Aksornphusitaphong $A$, Phupong $V$. Risk factors of early and late onset pre-eclampsia. Journal of Obstetrics and Gynaecology Research. 2013;39(3):627-31.

14. C. W. Chen, I. Z. Jaffe, and S. A. Karumanchi. Pre-eclampsia and cardio-vascular disease. Cardiovasc Res. 2014;101(4):579-86.

15. Yakasai IA, Morhason-Bello IO. Risk factors for pre-eclampsia among women at antenatal booking in Kano, Northern Nigeria. Healthcare in Low-resource Settings. 2013;1(1):12.
16. Prajapati SR, Maitra N. Prediction of pre-eclampsia by a combination of maternal history, uterine artery Doppler, and mean arterial pressure (a prospective study of 200 cases). The Journal of Obstetrics and Gynecology of India. 2013;63(1):32-6.

17. Kartasurya MI. Pre-eclampsia risk factors of pregnant women in Semarang, Indonesia. IJSBAR. 2015;22:31-7.

18. Anderson NH, McCowan LM, Fyfe EM, Chan EH, Taylor RS, Stewart AW, et al. The impact of maternal body mass index on the phenotype of pre-eclampsia: a prospective cohort study. BJOG: An International Journal of Obstetrics \& Gynaecology. 2012;119(5):589-95.

19. Lie RT, Rasmussen S, Brunborg H, Gjessing HK, Lie-Nielsen E, Irgens LM. Fetal and maternal contributions to risk of pre-eclampsia: population based study. Bmj. 1998;316(7141):1343.

20. Minassian C, Thomas SL, Williams DJ, Campbell O, Smeeth L. Acute maternal infection and risk of pre-eclampsia: a population-based case-control study. PloS one. 2013 Sep 3;8(9):e73047.

21. Torjusen $\mathrm{H}$, Brantsæter $A L$, Haugen M, Alexander J, Bakketeig LS, Lieblein G, et al. Reduced risk of pre-eclampsia with organic vegetable consumption: results from the prospective Norwegian Mother and Child Cohort Study. BMJ open. 2014;4(9):e006143.

22. Ganesh KS, Unnikrishnan B, Nagaraj K, Jayaram S. Determinants of Pre-eclampsia: A Case-control Study in a District Hospital in South India. Indian journal of community medicine: official publication of Indian Association of Preventive \& Social Medicine. 2010 Oct;35(4):502-5.

23. Conde-Agudelo A, Belizán JM. Risk factors for pre-eclampsia in a large cohort of Latin American and Caribbean women. BJOG: An International Journal of Obstetrics \& Gynaecology. 2000;107(1):75-83.

24. Luanni V.B, Erika Ota, Togoobaatar G, Rintaro M, and João Paulo S. Risk Factors of Pre-Eclampsia/Eclampsia and Its Adverse Outcomes in Low- and MiddleIncome Countries: A WHO Secondary Analysis. PLoS One. 2014;9(3):e91198.

25. Latha K, Judie A. Unveiling The Precedence of Risk Factors for Pre-Eclampsia: for Suitable Surveillance and Prophylaxis. IJSR- International Journal Of Scientific Research.2014;3(7):439-42.

26. Ishag Adam, AbdEIrahium D. Haggaz, Omer A. Mirghani, and Elhassan M. Elhassan. Placenta previa and Preeclampsia: analyses of 1645 cases at Medani maternity hospital, Sudan.Front Physiol. 2013;4:32.

27. Duckitt K, Harrington D. Risk factors for pre-eclampsia at antenatal booking: systematic review of controlled studies. Bmj. 2005;330(7491):565.

28. Lamminpää R, Vehviläinen-Julkunen K, Gissler M, Heinonen S. Preeclampsia complicated by advanced maternal age: a registry-based study on primiparous women in Finland 1997-2008. BMC pregnancy and childbirth. 2012;12(1):47.

29. Kirsten Duckitt, Deborah Harrington. Risk factors for pre-eclampsia at antenatal booking: systematic review of controlled studies. BMJ. 2005;330:565.

30. Lakshman R, Forouhi NG, Sharp SJ, Luben R, Bingham SA, Khaw KT, et al. Early age at menarche associated with cardiovascular disease and mortality. The Journal of Clinical Endocrinology \& Metabolism. 2009 Dec 1;94(12):4953-60.

31. Cerón-Mireles P, Harlow SD, Sanchez-Carrillo Cl, Nunez RM. Risk factors for pre-eclampsia/eclampsia among working women in Mexico City. Paediatric and perinatal epidemiology. 2001;15(1):40-6.

32. North RA, McCowan LM, Dekker GA, Poston L, Chan EH, Stewart AW, Black MA, Taylor RS, Walker JJ, Baker PN, Kenny LC. Clinical risk prediction for preeclampsia in nulliparous women: development of model in international prospective cohort. Bmj. 2011;342:d1875.

33. Walker JJ. Severe pre-eclampsia and eclampsia. Baillieres Best Pract Res Clin Obstet Gynaecol. 2000;14(1):57-71.

34. AgrawalS, Walia GK. Prevalence and risk factors for Pre-eclampsia in Indian women. J Womens Health, Issues Care. 2014;3:6.

Cite this article : Verma MK, Kapoor P, Yadav R, Manohar RK. Risk Factor Assessment for Preeclampsia: A Case Control Study. Int J Med Public Health. 2017;7(3):172-7. 